\title{
The Photochemistry of 1-Alkenyl-Substituted-1,2,3-Triazoles Leading to Formation of Pyrrole Derivatives
}

\author{
Nader A. Al-Jalal ${ }^{1}$, Nouria A. Al-Awadi ${ }^{1}$, Maher R. Ibrahim ${ }^{1} \&$ Mohamed H. Elnagdi ${ }^{1}$ \\ ${ }^{1}$ Department of Chemistry, Faculty of Science, Kuwait University, Safat, Kuwait \\ Correspondence: Nader A. Al-Jalal, Department of Chemistry, Faculty of Science, Kuwait University, P.O. Box \\ 5969, Safat 13060, Kuwait. Tel: 965-2498-7079. E-mail: nader.aljalal@ku.edu.kw
}

Received: July 23, 2013 Accepted: August 26, 2013 Online Published: October 14, 2013

doi:10.5539/ijc.v5n4p80 URL: http://dx.doi.org/10.5539/ijc.v5n4p80

\begin{abstract}
Irradiation of 1-alkenyl-substituted-1,2,3-triazoles 5a-d using $16 \mathrm{~W}$ low pressure mercury arc-lamp $(254 \mathrm{~nm})$ for $16 \mathrm{hrs}$ produced 3-dimethyamino- $1 H$-pyrrole derivatives 9a-d, together with 4-phenyl or (4-ethoxycarbonyl)-1H-1,2,3-triazoles 10a,b.
\end{abstract}

Keywords: photolysis, 1,2,3-triazoles, pyrrole, microwave

\section{Introduction}

Previous studies have shown that thermolytic and/or photolytic reactions of 1-substituted benzotriazole derivatives take place with elimination of $\mathrm{N}_{2}$ followed by subsequent ring closure of the resulting biradical intermediates to form heterocyclic products (Dib, Al-Awdi, Ibrahim, \& El-Desoqui, 2003, 2004; H. Al-Awadi, M. Ibrahim, Y. Ibrahim, \& N. Al-wadi, 2008; Maerky, Schmid, \& Hansen, 1979; Wender \& Cooper, 1986). These efficient processes have been described by Katritzky and his coworkers (Katritzky, Lan, Yang, \& Denisko, 1998). More recently we have reported the synthesis of indoles $\mathbf{2}$ and benzimidazoles $\mathbf{3}$ via photolysis of readily obtainable 1-substituted-1,2,3-benzotriazoles 1a,b (Scheme 1) (Al-Jalal, Al-Awadi, Ibrahim, \& Elnagdi, 2011a, 2011b).

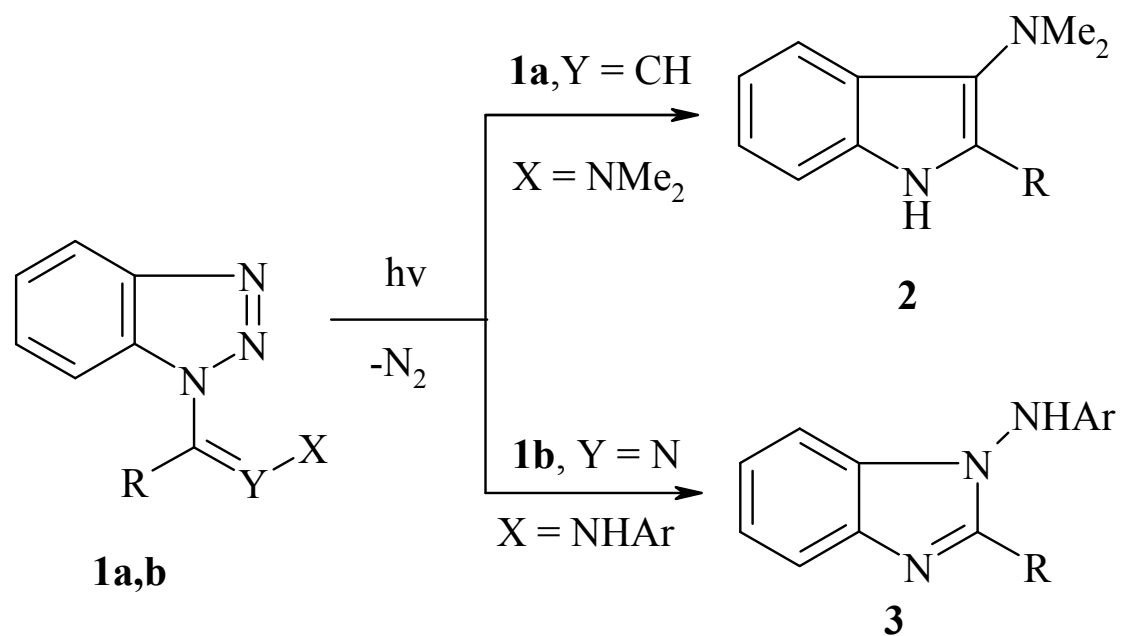

Scheme 1. Photolysis of 1-substituted benzotriazoles $1 \mathrm{a}, \mathrm{b}$ to indoles and benzimidazoles

However, literature survey indicated that little investigation have been made on thermolytic and/or photolytic behavior of 1-alkenyl-substituted-1,2,3-triazoles (Boyer \& Silvarajan, 1969; Burgess, Carithers, \& McCullagh, 1968; Michell \& Rees, 1987; Ogata, Takaji, \& Hayashi, 1977; Silvarajan \& Boyer, 1972; Wender \& Cooper, 1969). In the light of the established pharmaceutical activity of 3-dimethylaminopyrrole which is reported as an analgesics and anticonvulsants agent (Bellina \& Rossi, 2006; Rochais, Lisowski, Dallemagne, \& Rault, 2004; 
Liebscher et al., 1992) we became interested in developing photoinduced synthetic route to such compounds via photolysis of suitably 1-substituted-1,2,3-triazole.

In this work we report the first successful photochemical conversion of various 1-alkenylsubstituted-1,2,3-triazoles into polysubstituted pyrrole derivatives by elimination of $\mathrm{N}_{2}$ followed by ring closure of the resulting biradical intermediates.

\section{Experimental}

General: Melting points were recorded on a Gallenkamp apparatus. IR spectra were recorded in $\mathrm{KBr}$ disks on a Perkin Elmer System 2000 FT-IR spectrophotometer. ${ }^{1} \mathrm{H}$ - and ${ }^{13} \mathrm{C}$ - NMR spectra were recorded on a Bruker DPX $400 \mathrm{MHz}$ NMR spectrometer with proton spectra measured at $400 \mathrm{MHz}$ and carbon spectra at $100 \mathrm{MHz}$. Mass spectra were measured on a VG Auto-spec-Q (high resolution, high performance, tri-sector GC/MS/MS) and with LCMS using Agilent 1100 series LC/MSD with an API-ES/APCI ionization mode. Microanalyses were performed on LECO CH NS-932 Elemental Analyzer. The UV-visible absorption spectra were scanned using Varian Cary 5 instrument in the wave length range 200-450 Explorer. Microwave experiments were carried out using a CEM Corporation, NC, USA microwave apparatus. X-ray analysis was performed using a Rigaku Rapid II and Bruker X8 Prospector diffractmeter. A photochemical reactors limited fitted with a $16 \mathrm{~W}$ low pressure mercury arc-lamp was used for the irradiation.

\subsection{Click Synthesis of 1-Alkyl Substituted-1,2,3-Triazoles $4 a-f$}

General procedure: A mixture of terminal alkynes ( $2.50 \mathrm{~g}, \approx 25 \mathrm{mmole})$, sodium azide ( $1.95 \mathrm{~g}, 30 \mathrm{mmole})$, alkyl halide derivatives $(25 \mathrm{mmol})$, copper sulfate pentahydrate $(0.6225 \mathrm{~g}, 0.01$ equiv) and sodium ascorbate $(0.99 \mathrm{~g}$, 0.02 equiv) in a mixture solvent of tertiary butanol and water $(1: 1 \mathrm{v} / \mathrm{v}, 50 \mathrm{ml})$, was stirred at room temperature for 3-12 hrs. After the color of the mixture was changed to yellow brown it was poured on to ice water $(150 \mathrm{ml})$, filtered, washed with water to give compounds $4 \mathbf{a}-\mathbf{f}$.

\subsubsection{1-(4-Phenyl-1,2,3-Triazol-1-yl)Propan-2-One 4a}

Colorless solid from ethanol, yield $4.0 \mathrm{~g}(80 \%)$, mp. 142-144 ${ }^{\circ} \mathrm{C}$. LCMS $(\mathrm{m} / \mathrm{z})=202(\mathrm{M}+1) .{ }^{1} \mathrm{H}$ NMR $(400$ $\left.\mathrm{MHz}, \mathrm{CDCl}_{3}\right): \delta 8.89-8.86(\mathrm{~m}, 3 \mathrm{H}), 7.46(\mathrm{td}, 2 \mathrm{H}, J=7.4,1.6 \mathrm{~Hz}), 7.37(\mathrm{tt}, 1 \mathrm{H}, J=7.6,1.6 \mathrm{~Hz}), 5.29(\mathrm{~s}, 2 \mathrm{H})$, $2.32(\mathrm{~s}, 3 \mathrm{H}) .{ }^{13} \mathrm{C}$ NMR $\left(100 \mathrm{MHz}, \mathrm{DMSO}-\mathrm{d}_{6}\right): \delta 200.9,146.2,130.7,128.9,127.9,125.1,122.7,58.4,27.1$ (HRMS $=201.0896$, requires $\mathrm{C}_{11} \mathrm{H}_{11} \mathrm{~N}_{3} \mathrm{O}$ 201.0902).

\subsubsection{4-Phenyl-1,2,3-Triazol-1-yl-acetic Acid Isopropyl Ester 4b}

Colorless solid from benzene, yield $4.0 \mathrm{~g}(78 \%), \mathrm{mp} .130-132{ }^{\circ} \mathrm{C}$. MS: $m / z(\%)=245\left(\mathrm{M}^{+}, 30\right), 175(100), 116$ (100). IR $\left(\mathrm{KBr}, \mathrm{cm}^{-1}\right): 3087,2987,1789,1463,1443,1379,1259,1105,1045,765,694 .{ }^{1} \mathrm{H}$ NMR $(400 \mathrm{MHz}$, $\left.\mathrm{CDCl}_{3}\right): \delta 7.94(\mathrm{~s}, 1 \mathrm{H}), 7.88(\mathrm{dd}, 2 \mathrm{H}, J=7.8,1.2 \mathrm{~Hz}), 7.46(\mathrm{t}, 2 \mathrm{H}, J=7.6 \mathrm{~Hz}), 7.38(\mathrm{tt}, 1 \mathrm{H}, J=7.8,1.2 \mathrm{~Hz})$, $5.20(\mathrm{~s}, 2 \mathrm{H}), 5.16(\mathrm{sex}, 1 \mathrm{H}, J=6.4 \mathrm{~Hz}), 1.32\left(\mathrm{~d}, 6 \mathrm{H}, J=6.4 \mathrm{~Hz}, 2 \mathrm{CH}_{3}\right) .{ }^{13} \mathrm{C}$ NMR $\left(100 \mathrm{MHz}, \mathrm{CDCl}_{3}\right): \delta 165.8$, $148.3,130.4,128.9,128.3,125.8,120.9,70.6,51.2,21.7(2 \mathrm{C})\left(\mathrm{HRMS}=245.1164\right.$, requires $\mathrm{C}_{13} \mathrm{H}_{15} \mathrm{~N}_{3} \mathrm{O}_{2}$ 245.1157).

\subsubsection{1-(2-Oxo-2-phenyl-ethyl)-1H-1,2,3-triazole-4-carboxylic Acid Ethyl Ester 4c}

Colorless solid from ethanol, yield $4.5 \mathrm{~g}(69 \%)$, mp.170-172 ${ }^{\circ} \mathrm{C}$. MS: $m / z(\%)=259\left(\mathrm{M}^{+}, 10\right), 202(50) .{ }^{1} \mathrm{H}$ NMR $\left(400 \mathrm{MHz}, \mathrm{CDCl}_{3}\right): \delta 8.31(\mathrm{~s}, 1 \mathrm{H}), 8.02(\mathrm{dd}, 2 \mathrm{H}, J=7.6,1.2 \mathrm{~Hz}), 7.72(\mathrm{t}, 1 \mathrm{H}, J=7.6 \mathrm{~Hz}), 7.59(\mathrm{t}, 2 \mathrm{H}, J=7.6$ $\mathrm{Hz}), 5.95(\mathrm{~s}, 2 \mathrm{H}), 4.46(\mathrm{q}, 2 \mathrm{H}, J=7.2 \mathrm{~Hz}), 1.44(\mathrm{t}, 3 \mathrm{H}, J=7.2 \mathrm{~Hz}) .{ }^{13} \mathrm{C}$ NMR $\left(100 \mathrm{MHz}, \mathrm{CDCl}_{3}\right): \delta 189.4$, $160.6,140.7,134.9,133.6,129.5,129.3,128.2,61.4,55.5,14.5\left(\mathrm{HRMS}=259.0951\right.$, requires $\mathrm{C}_{13} \mathrm{H}_{13} \mathrm{~N}_{3} \mathrm{O}_{3}$ 259.0957).

\subsubsection{4-Phenyl-1,2,3-Triazol-1-yl-acetic Acid Methyl Ester 4d}

Yellow solid from ethanol, yield $7.5 \mathrm{~g}(88 \%)$, mp. $82-83{ }^{\circ} \mathrm{C}$ (lit.mp $81-82{ }^{\circ} \mathrm{C}$, Kumar, Patel, \& Reddy, 2009).

\subsubsection{1-Allyl-4-phenyl-1 $H$-1,2,3-triazole $4 \mathbf{e}$}

Colorless solid from benzene, yield $3.6 \mathrm{~g}(78 \%)$, mp.110-112 ${ }^{\circ} \mathrm{C}$ (lit.mp. 112-113 ${ }^{\circ} \mathrm{C}$, Kidwai \& Jain, 2011).

2.1.6 Trans 1-(2-Ethoxycarbonylvinyl)-1H-1,2,3-Triazole-4-Carboxylic Acid Ethyl Ester $\mathbf{4 f}$

This compound was prepared from ethyl propiolate $(2.0 \mathrm{~g}, 20.0 \mathrm{mmol})$ and sodium azide $(0.78 \mathrm{~g}, 12.0 \mathrm{mmol})$ after stirring at room temperature for $3 \mathrm{hrs}$ at the same condition of general procedure as colorless crystals from benzene, $\left(\mathrm{R}_{\mathrm{f}}\right.$ 0.5, EtOAc: petroleum b.p. $\left.60-80,1: 2 \mathrm{v} / \mathrm{v}\right)$, yield $1.2 \mathrm{~g}(50 \%), \mathrm{mp} .93-95{ }^{\circ} \mathrm{C}$. MS: $\mathrm{m} / \mathrm{z}(\%)=239$ $\left(\mathrm{M}^{+}, 10\right), 194(60), 138(100)$. IR $\left(\mathrm{KBr}, \mathrm{cm}^{-1}\right)$ : 3103, 3051, 2980, 1735, 1713, 1663, 1539, 1447, 1373, 1305, $1210,1157,1049,961,864,781 .{ }^{1} \mathrm{H}$ NMR $\left(400 \mathrm{MHz}, \mathrm{CDCl}_{3}\right): \delta 8.38(\mathrm{~s}, 1 \mathrm{H}), 8.23(\mathrm{~d}, 1 \mathrm{H}, J=14.4 \mathrm{~Hz}), 6.67(\mathrm{~d}$, 
$1 \mathrm{H}, J=14.4 \mathrm{~Hz}), 4.47(\mathrm{q}, 2 \mathrm{H}, J=7.2 \mathrm{~Hz}), 4.32(\mathrm{q}, 2 \mathrm{H}, J=7.2 \mathrm{~Hz}), 1.45(\mathrm{t}, 3 \mathrm{H}, J=7.2 \mathrm{~Hz}), 1.37(\mathrm{t}, 3 \mathrm{H}, J=7.2$ $\mathrm{Hz}) .{ }^{13} \mathrm{C} \mathrm{NMR}\left(100 \mathrm{MHz}, \mathrm{CDCl}_{3}\right): \delta 164.6,160.0,141.1,135.3,126.0,113.0,61.8,61.5,14.3,14.2(\mathrm{HRMS}=$ 239.0900, requiers $\left.\mathrm{C}_{10} \mathrm{H}_{13} \mathrm{~N}_{3} \mathrm{O}_{4} 239.0906\right)$.

\subsubsection{Cis 1-(2-Ethoxycarbonylvinyl)-1H-1,2,3-Triazole-4-Carboxylic Acid Ethyl Ester 4f}

White solid from petroleum b.p. $60-80,\left(\mathrm{R}_{\mathrm{f}} 0.65\right.$, EtOAc: petroleum b.p. $\left.60-80{ }^{\circ} \mathrm{C}, 1: 2 \mathrm{v} / \mathrm{v}\right)$, yield $0.5 \mathrm{~g}(20 \%)$, mp. 66-68 ${ }^{\circ} \mathrm{C}$. MS: $m / z(\%)=239\left(\mathrm{M}^{+}, 10\right), 194(60), 138(100)$. IR (KBr, cm $\left.{ }^{-1}\right): 3103,3052,2980,1735,1713$, 1663, 1539, 1447, 1373, 1304, 1208, 1157, 1048, 961, 781. ${ }^{1} \mathrm{H}$ NMR $\left(400 \mathrm{MHz}, \mathrm{CDCl}_{3}\right): \delta 9.69(\mathrm{~s}, 1 \mathrm{H}), 7.65(\mathrm{~d}$, $1 \mathrm{H}, J=10.8 \mathrm{~Hz}), 5.83(\mathrm{~d}, 1 \mathrm{H}, J=10.8 \mathrm{~Hz}), 4.46(\mathrm{q}, 2 \mathrm{H}, J=7.0 \mathrm{~Hz}), 4.29(\mathrm{q}, 2 \mathrm{H}, J=7.0 \mathrm{~Hz}), 1.44(\mathrm{t}, 3 \mathrm{H}, J=$ $7.0 \mathrm{~Hz}), 1.36(\mathrm{t}, 3 \mathrm{H}, J=7.0 \mathrm{~Hz}) .{ }^{13} \mathrm{C} \mathrm{NMR}\left(100 \mathrm{MHz}, \mathrm{CDCl}_{3}\right): \delta 163.7,160.2,140.2,133.0,130.0,109.6,61.5$ (2C), 14.3, 14.0 (HRMS = 239.0900, requires $\left.\mathrm{C}_{10} \mathrm{H}_{13} \mathrm{~N}_{3} \mathrm{O}_{4} 239.0906\right)$.

\subsection{Synthesis of 1-Alkenyl substituted-1,2,3-Triazole Derivatives $\mathbf{5 a - d}$}

General procedure: A mixture of each of compounds 4 a-d $(10 \mathrm{mmol})$ and dimethylformamidedimethyleacetal (DMF-DMA) $(3 \mathrm{ml}, 25 \mathrm{mmol})$ in xylene $(3 \mathrm{ml})$ was introduced in a microwave oven and irradiated at $150{ }^{\circ} \mathrm{C}$ for 3 minutes. The mixture was cooled and poured on petroleum ether p.b. $60-80{ }^{\circ} \mathrm{C}(50 \mathrm{ml})$, filtered, and crystallized from ethanol to give compounds 5a-d.

\subsubsection{4-Dimethylamino-3-(4-Phenyl-1,2,3-Triazol-1-yl)but-3-en-2-one 5a}

White solid, yield $2.0 \mathrm{~g}(78 \%)$, mp. $160-162{ }^{\circ} \mathrm{C} . \mathrm{MS}: \mathrm{m} / z(\%)=256\left(\mathrm{M}^{+}, 5\right), 228(100), 185(25) . \mathrm{IR}\left(\mathrm{KBr}, \mathrm{cm}^{-1}\right)$ :

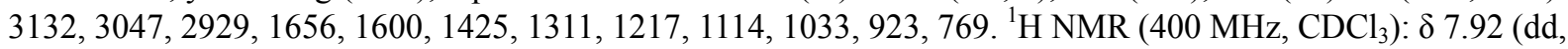
$2 \mathrm{H}, J=7.6,1.2 \mathrm{~Hz}), 7.87(\mathrm{~s}, 1 \mathrm{H}), 7.73(\mathrm{~s}, 1 \mathrm{H}), 7.48(\mathrm{t}, 2 \mathrm{H}, J=7.4 \mathrm{~Hz}), 7.39$ (t, $1 \mathrm{H}, J=7.8 \mathrm{~Hz}), 3.20(\mathrm{~s}, 3 \mathrm{H}$, $\left.\mathrm{CH}_{3}\right), 2.35\left(\mathrm{~s}, 3 \mathrm{H}, \mathrm{CH}_{3}\right), 1.91\left(\mathrm{~s}, 3 \mathrm{H}, \mathrm{CH}_{3}\right) .{ }^{13} \mathrm{C} \mathrm{NMR}\left(100 \mathrm{MHz}, \mathrm{CDCl}_{3}\right): \delta 192.3,147.9,146.4,130.2,129.0$, $128.4,125.7,125.1,108.2,47.9,36.7,24.6$ (HRMS $=256.1319$, requires $\left.\mathrm{C}_{14} \mathrm{H}_{16} \mathrm{~N}_{4} \mathrm{O} 256.1324\right)$.

\subsubsection{3-Dimethylamino-2-(4-Phenyl-1,2,3-Triazol-1-yl)acrylic Acid Isopropyl Ester 5b}

White solid, yield $2.1 \mathrm{~g}(70 \%)$, mp. $155-156^{\circ} \mathrm{C} . \mathrm{MS}: \mathrm{m} / z(\%)=300\left(\mathrm{M}^{+}, 5\right), 229(100), 201(25) . \mathrm{IR}\left(\mathrm{KBr}, \mathrm{cm}^{-1}\right)$ : 3091, 2976, 2967, 1694, 1623, 1465, 1428, 1348, 1285, 1226, 1108, 1085, 1046, 768, 694. ${ }^{1} \mathrm{H}$ NMR (400 MHz, $\left.\mathrm{CDCl}_{3}\right): \delta 7.90(\mathrm{dd}, 2 \mathrm{H}, J=7.6,1.2 \mathrm{~Hz}), 7.86(\mathrm{~s}, 1 \mathrm{H}), 7.63(\mathrm{~s}, 1 \mathrm{H}), 7.46(\mathrm{t}, 2 \mathrm{H}, J=7.6 \mathrm{~Hz}), 7.36(\mathrm{t}, 1 \mathrm{H}, J=7.8$

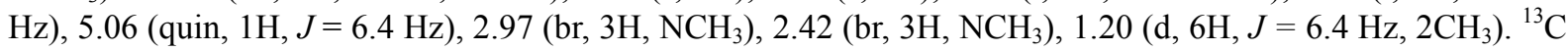
NMR (100 MHz, $\left.\mathrm{CDCl}_{3}\right): \delta 165.3,147.0,146.5,130.5,128.9,128.2,125.9,125.7,97.3,67.7,47.6,36.9,22.0$ (2C) (HRMS $=300.1580$, requires $\left.\mathrm{C}_{16} \mathrm{H}_{20} \mathrm{~N}_{4} \mathrm{O}_{2} 300.1586\right)$.

2.2.3 1-(1-Benzoyl-2-Dimethylamino-vinyl)-1H-1,2,3-Triazole-4-Carboxylic Acid Ethyl Ester 5c

Colorless solid, yield $2.3 \mathrm{~g}(73 \%)$, mp.132-134 ${ }^{\circ} \mathrm{C}$. LCMS: $(m / z)=315(\mathrm{M}+1)$. MS: $m / z(\%)=314\left(\mathrm{M}^{+}, 15\right)$, 286 (15), 213 (40), 105 (100\%). IR (KBr, cm $\left.{ }^{-1}\right): 3132,3047,2929,1656,1600,1425,1311,1217,1114,1033$, 923, 769. ${ }^{1} \mathrm{H}$ NMR $\left(400 \mathrm{MHz}, \mathrm{CDCl}_{3}\right): \delta 8.13(\mathrm{~s}, 1 \mathrm{H}), 7.56(\mathrm{~s}, 1 \mathrm{H}), 7.47(\mathrm{~d}, 2 \mathrm{H}, J=7.6 \mathrm{~Hz}), 7.44(\mathrm{t}, 1 \mathrm{H}, J=7.6$ $\mathrm{Hz}), 7.39-7.35$ (m, 2H), 4.43 (q, 2H, $J=7.2 \mathrm{~Hz}), 3.17$ (br, 3H, NCH$), 2.38\left(\mathrm{br}, 3 \mathrm{H}, \mathrm{NCH}_{3}\right), 1.42(\mathrm{t}, 3 \mathrm{H}, J=7.2$ Hz). $\left.{ }^{13} \mathrm{C} \mathrm{NMR} \mathrm{(100} \mathrm{MHz,} \mathrm{CDCl}_{3}\right): \delta 188.7,160.7,150.2,140.0,138.5,133.2,130.7,128.4,127.9,108.3,61.4$, 48.2, 38.0, 14.3 (HRMS = 314.1373, requires $\mathrm{C}_{16} \mathrm{H}_{18} \mathrm{~N}_{4} \mathrm{O}_{3} 314.1379$ ).

\subsubsection{3-Dimethylamino-2-(4-Phenyl-1,2,3-Triazol-1-yl)acrylic Acid Methyl Ester 5d}

Colorless solid, mp.118-120 ${ }^{\circ} \mathrm{C}$, yield $2.0 \mathrm{~g}(73 \%)$. MS: $m / z(\%)=272\left(\mathrm{M}^{+}, 5\right), 244(50), 229(100) . \mathrm{IR}(\mathrm{KBr}$, $\left.\mathrm{cm}^{-1}\right): 3133,2948,1698,1640,1468,1430,1402,1327,1294,1223,1158,1109,1082,1023,764 .{ }^{1} \mathrm{H} \mathrm{NMR}(400$ $\left.\mathrm{MHz}_{\mathrm{CDCl}}\right): \delta 7.88(\mathrm{~d}, 2 \mathrm{H}, J=7.6 \mathrm{~Hz}), 7.84(\mathrm{~s}, 1 \mathrm{H}), 7.65(\mathrm{~s}, 1 \mathrm{H}), 7.44(\mathrm{t}, 2 \mathrm{H}, J=7.8 \mathrm{~Hz}), 7.34(\mathrm{t}, 1 \mathrm{H}, J=7.2$ $\mathrm{Hz}), 3.66\left(\mathrm{~s}, 3 \mathrm{H}, \mathrm{OCH}_{3}\right), 3.13\left(\mathrm{br}, 3 \mathrm{H}, \mathrm{NCH}_{3}\right), 2.34\left(\mathrm{br}, 3 \mathrm{H}, \mathrm{NCH}_{3}\right) .{ }^{13} \mathrm{C} \mathrm{NMR}\left(100 \mathrm{MHz}, \mathrm{CDCl}_{3}\right): \delta 166.2$, 146.9, 146.1, 130.4, 128.8, 128.2, 125.7, 125.5, 96.7, 51.6, 47.6, $36.2\left(\mathrm{HRMS}=272.1268\right.$, requires $\mathrm{C}_{14} \mathrm{H}_{16} \mathrm{~N}_{4} \mathrm{O}_{2}$ 272.1273).

\subsection{Synthesis of 1-Arylhydrazono-1,2,3-Triazole Derivatives 6a-c}

General procedure: To a cooled solution $\left(0{ }^{\circ} \mathrm{C}\right)$ of compound $4 \mathbf{a}(2.01 \mathrm{~g}, 10 \mathrm{mmol})$, sodium acetate $(1.64 \mathrm{~g}, 20$ $\mathrm{mmol})$ in ethanol $(100 \mathrm{ml})$ was gradually added with stirring in about $30 \mathrm{~min}$ cooled solution of appropriate aromatic diazonium chloride $(10 \mathrm{mmol})$. The mixture was stirred for 24 hours at room temperature. The yellow solid so formed was filtered and crystallized from ethanol to give compounds $\mathbf{6 a - c}$.

\subsubsection{1-(Phenylhydrazono)-1-(4-Phenyl-1,2,3-Triazol-1-yl)propan-2-one 6a}

Yield 2.3 g (75\%), mp.155-156 ${ }^{\circ}$ C.MS: $m / z(\%)=305\left(\mathrm{M}^{+}, 5\right), 277(40), 92(100)$. IR (KBr, $\left.\mathrm{cm}^{-1}\right): 3179,3162$, $3038,1668,1562,1552,1510,1378,1355,1257,1238,1210,1142,1078,1012,763,690 .{ }^{1} \mathrm{H} \mathrm{NMR}(400 \mathrm{MHz}$, $\left.\mathrm{CDCl}_{3}\right): \delta 12.23(\mathrm{~s}, 1 \mathrm{H}), 9.05(\mathrm{~s}, 1 \mathrm{H}), 7.95(\mathrm{dd}, 2 \mathrm{H}, J=8.4,1.2 \mathrm{~Hz}), 7.49(\mathrm{t}, 2 \mathrm{H}, J=7.6 \mathrm{~Hz}), 7.44-7.37(\mathrm{~m}, 5 \mathrm{H})$, 
7.16-7.12 (m, 1H), $2.73(\mathrm{~s}, 3 \mathrm{H}) .{ }^{13} \mathrm{C}$ NMR (100 MHz, $\left.\mathrm{CDCl}_{3}\right): \delta$ 190.6, 146.1, 142.0, 129.6, 129.0, $128.8(2 \mathrm{C})$, 126.1, 124.2, 123.9, 120.8, 115.1, 26.1. Anal. Calc. for $\mathrm{C}_{17} \mathrm{H}_{15} \mathrm{~N}_{5} \mathrm{O}$ (305.34): C, 66.87; H, 4.95; N, 22.94. Found: C, 66.79; H, 5.01; N, 22.84 .

\subsubsection{1-p-Chlorophenylhydrazono-1-(4-Phenyl-1,2,3-Triazol-1-yl)propan-2-one $\mathbf{6 b}$}

Yield $2.5 \mathrm{~g}(73 \%)$, mp. $158-160{ }^{\circ} \mathrm{C}$. MS: $m / z(\%)=339\left(\mathrm{M}^{+}, 10\right), 311(40), 126(100)$. IR $\left(\mathrm{KBr}, \mathrm{cm}^{-1}\right): 3271$, $3149,3058,3038,1673,1564,1488,1397,1258,1231,1083,1011,821,760,691 .{ }^{1} \mathrm{H}$ NMR $\left(400 \mathrm{MHz}, \mathrm{CDCl}_{3}\right)$ : $\delta 12.26(\mathrm{~s}, 1 \mathrm{H}), 9.04(\mathrm{~s}, 1 \mathrm{H}), 7.92(\mathrm{dd}, 2 \mathrm{H}, J=8.4,1.6 \mathrm{~Hz}), 7.48(\mathrm{dt}, 2 \mathrm{H}, J=8.0,1.2 \mathrm{~Hz}), 7.41(\mathrm{tt}, 1 \mathrm{H}, J=8.0$, $1.2 \mathrm{~Hz}), 7.36(\mathrm{~d}, 2 \mathrm{H}, J=8.0 \mathrm{~Hz}), 7.30(\mathrm{~d}, 2 \mathrm{H}, J=8.0 \mathrm{~Hz}), 2.71(\mathrm{~s}, 3 \mathrm{H}) .{ }^{13} \mathrm{C}$ NMR $\left(100 \mathrm{MHz}, \mathrm{CDCl}_{3}\right): \delta 190.5$, 146.1, 140.6, 129.7, 129.5, 129.1, 129.0, 128.8, 126.0, 124.1, 120.8, 116.2, 26.1. Anal Calc. for $\mathrm{C}_{17} \mathrm{H}_{14} \mathrm{ClN}_{5} \mathrm{O}$ (339.79): C, 60.09; H, 4.15; N, 20.61. Found: C, 60.02; H, 4.24; N, 20.49.

\subsubsection{1-(4-Phenyl-1,2,3-Triazol-1-yl)-1-p-Tolylhydrazono-propan-2-one $\mathbf{6 c}$}

Yield $2.4 \mathrm{~g}(75 \%), \mathrm{mp} .164-166^{\circ} \mathrm{C}$. MS: $m / z(\%)=319\left(\mathrm{M}^{+}, 5\right), 291(70), 106(100)$. IR $\left(\mathrm{KBr}, \mathrm{cm}^{-1}\right): 3268,3171$, $3038,1661,1552,1506,1402,1265,1145,1013,818,760,687 .{ }^{1} \mathrm{H}$ NMR $\left(400 \mathrm{MHz}, \mathrm{CDCl}_{3}\right): \delta 12.21(\mathrm{~s}, 1 \mathrm{H})$, $9.07(\mathrm{~s}, 1 \mathrm{H}), 7.95(\mathrm{dd}, 2 \mathrm{H}, J=8.4,1.2 \mathrm{~Hz}), 7.50(\mathrm{t}, 2 \mathrm{H}, J=7.6 \mathrm{~Hz}), 7.42(\mathrm{t}, 1 \mathrm{H}, J=7.6 \mathrm{~Hz}), 7.29(\mathrm{~d}, 2 \mathrm{H}, J=8.4$ $\mathrm{Hz}), 7.23(\mathrm{~d}, 2 \mathrm{H}, J=8.4 \mathrm{~Hz}), 2.73(\mathrm{~s}, 3 \mathrm{H}), 2.38(\mathrm{~s}, 3 \mathrm{H}) .{ }^{13} \mathrm{C} \mathrm{NMR}\left(100 \mathrm{MHz}, \mathrm{CDCl}_{3}\right): \delta 190.4,146.0,139.7$, 133.9, 130.2, 129.7, 129.0, 128.7, 126.0, 123.7, 120.8, 115.1, 26.0, 20.9. Anal Calc. for $\mathrm{C}_{18} \mathrm{H}_{17} \mathrm{~N}_{5} \mathrm{O}(319.37)$ : C, $67.70 ; \mathrm{H}, 5.37 ; \mathrm{N}, 21.93$. Found: $\mathrm{C}, 67.72 ; \mathrm{H}, 5.34 ; \mathrm{N}, 21.80$.

\subsection{Photolysis of Compounds $4 \boldsymbol{e}, \boldsymbol{f}, \mathbf{5 a - d}$ and $\mathbf{6} \boldsymbol{a}-\boldsymbol{c}$}

Irradiation using ( $16 \mathrm{w})$ low pressure mercury arc-lamp. Each of the substrates 4e,f, 5a-d and 6a-c (1.0 mmol) was dissolved in acetonitrile $(25 \mathrm{~mL})$ in quartz tubes and irradiated using $(16 \mathrm{~W})$ low pressure mercury arc-lamp for $16 \mathrm{hrs}$ at room temperature. The progress of the reaction was monitored by TLC and the formation of products was detected with LCMS. The solvent was removed in vacuo and the resulting residue was subjected to column chromatography on silica gel using ethyl acetate/petroleum ether b.p. $60-80^{\circ} \mathrm{C}$ the $\%$ yield in (Table 1).

\subsubsection{2-Acetyl-3-Dimethylamino-4-Phenyl-1 $H$-Pyrrole 9a}

Yellow solid, $\left(\mathrm{R}_{\mathrm{f}} 0.55\right.$, EtOAc: petroleum b.p. $\left.60-80{ }^{\circ} \mathrm{C}, 1: 4 \mathrm{v} / \mathrm{v}\right)$. mp. $158-160{ }^{\circ} \mathrm{C} . \mathrm{MS}: \mathrm{m} / z(\%)=228\left(\mathrm{M}^{+}, 100\right)$, 211 (55), 169 (30). IR (KBr, $\left.\mathrm{cm}^{-1}\right): 3276,3070,2927,1614,1550,1458,1398,1286,1122,973,919,763 .{ }^{1} \mathrm{H}$ NMR $\left(400 \mathrm{MHz}, \mathrm{CDCl}_{3}\right): \delta 9.39(\mathrm{br}, 1 \mathrm{H}, \mathrm{NH}), 7.40-7.28(\mathrm{~m}, 5 \mathrm{H}), 6.89(\mathrm{~d}, 1 \mathrm{H}, J=3.6 \mathrm{~Hz}), 2.79(\mathrm{~s}, 6 \mathrm{H}$, $\mathrm{N}\left(\mathrm{CH}_{3}\right)_{2}, 2.61\left(\mathrm{~s}, 3 \mathrm{H}, \mathrm{CH}_{3}\right) .{ }^{13} \mathrm{C} \mathrm{NMR}\left(100 \mathrm{MHz}, \mathrm{CDCl}_{3}\right): \delta 188.5,135.1,129.6(2 \mathrm{C}), 128.2,127.0,125.6$, 123.7, 122.6, 45.6 (2C), $26.4\left(\mathrm{HRMS}=228.1257\right.$, requires $\left.\mathrm{C}_{14} \mathrm{H}_{16} \mathrm{~N}_{2} \mathrm{O} 228.1263\right)$.

\subsubsection{3-Dimethylamino-4-Phenyl-1 $H$-Pyrrole-2-Carboxylic Acid Isopropyl Ester 9b}

Colorless solid, $\left(\mathrm{R}_{\mathrm{f}} 0.70\right.$, EtOAc: petroleum b.p. $\left.60-80{ }^{\circ} \mathrm{C}, 1: 4 \mathrm{v} / \mathrm{v}\right)$. mp. $150-152{ }^{\circ} \mathrm{C}$. MS: $m / z(\%)=272\left(\mathrm{M}^{+}\right.$, 90), 212 (100), 169 (55). IR (KBr, cm $\left.{ }^{-1}\right): 3476,3088,2986,1753,1560,1465,1378,1334,1223,1191,1106$, 1082, 846, 766. ${ }^{1} \mathrm{H}$ NMR (400 MHz, $\left.\mathrm{CDCl}_{3}\right): \delta 8.82(\mathrm{br}, 1 \mathrm{H}), 7.55$ (d, $\left.2 \mathrm{H}, J=7.6 \mathrm{~Hz}\right), 7.38(\mathrm{t}, 2 \mathrm{H}, J=7.6 \mathrm{~Hz})$, $7.29(\mathrm{t}, 1 \mathrm{H}, J=7.4 \mathrm{~Hz}), 6.90(\mathrm{~d}, 1 \mathrm{H}, J=3.2 \mathrm{~Hz}), 5.31-5.25(\mathrm{~m}, 1 \mathrm{H}), 2.85\left(\mathrm{~s}, 6 \mathrm{H}, \mathrm{N}\left(\mathrm{CH}_{3}\right)_{2}, 1.39(\mathrm{~s}, 6 \mathrm{H}) .{ }^{13} \mathrm{C}\right.$ $\operatorname{NMR}\left(100 \mathrm{MHz}, \mathrm{CDCl}_{3}\right): \delta 159.7,128.3,128.2,126.3,126.2,125.3,122.3,119.7,115.8,67.6,44.2,34.4,22.3$ (2C) $\left(\mathrm{HRMS}=272.1519\right.$, requires $\left.\mathrm{C}_{16} \mathrm{H}_{20} \mathrm{~N}_{2} \mathrm{O}_{2} 272.1525\right)$.

\subsubsection{5-Benzoyl-3-Dimethylamino-1H-Pyrrole-3-Carboxylic Acid Ethyl Ester 9c}

Yellow solid, $\left(\mathrm{R}_{\mathrm{f}} 0.95\right.$, EtOAc: petroleum b.p. $\left.60-80,1: 4 \mathrm{v} / \mathrm{v}\right) . \mathrm{mp} .138-140{ }^{\circ} \mathrm{C} . \mathrm{LCMS}=287(\mathrm{M}+1) . \mathrm{MS}: \mathrm{m} / \mathrm{z}$ $(\%)=286\left(\mathrm{M}^{+}, 100\right), 269(60), 225(35)$. IR $\left(\mathrm{KBr}, \mathrm{cm}^{-1}\right): 3262,2979,2928,1714,1600,1534,1446,1401,1374$, 1273, 1184, 1093, 1032, 736. ${ }^{1} \mathrm{H}$ NMR $\left(400 \mathrm{MHz}, \mathrm{CDCl}_{3}\right): \delta 9.30(\mathrm{br}, 1 \mathrm{H}, \mathrm{NH}), 7.71(\mathrm{~d}, 2 \mathrm{H}, J=7.8 \mathrm{~Hz}), 7.56(\mathrm{~s}$, $1 \mathrm{H}), 7.54(\mathrm{t}, 1 \mathrm{H}, J=7.4 \mathrm{~Hz}), 7.47(\mathrm{t}, 2 \mathrm{H}, J=7.8 \mathrm{~Hz}), 4.30(\mathrm{q}, 2 \mathrm{H}, J=7.2 \mathrm{~Hz}), 2.61\left(\mathrm{~s}, 6 \mathrm{H}, 2 \mathrm{CH}_{3}\right), 1.37(\mathrm{t}, 3 \mathrm{H}, J$ $=7.2 \mathrm{~Hz}) \cdot{ }^{13} \mathrm{C}$ NMR $\left(100 \mathrm{MHz}, \mathrm{CDCl}_{3}\right): \delta 185.5,163.4,146.3,139.4,131.6,129.6,128.6,128.1,122.9,111.4$, $59.8,44.1(2 \mathrm{C}), 14.5$ (HRMS $=286.1314$, requires $\mathrm{C}_{16} \mathrm{H}_{18} \mathrm{~N}_{2} \mathrm{O}_{3}$ 286.1317).

\subsubsection{3-Dimethylamino-4-Phenyl-1H-Pyrrole-2-Carboxylic Acid Methyl Ester 9d}

Colorless solid, $\left(\mathrm{R}_{\mathrm{f}}\right.$ 0.9, EtOAc: petroleum b.p. $\left.60-80,1: 3 \mathrm{v} / \mathrm{v}\right) . \mathrm{mp} .142-144^{\circ} \mathrm{C} . \mathrm{LCMS}=245(\mathrm{M}+1) . \mathrm{MS}: \mathrm{m} / z$ $(\%)=244\left(\mathrm{M}^{+}, 45\right), 229(100), 201(25)$. IR $\left(\mathrm{KBr}, \mathrm{cm}^{-1}\right): 3329,2957,2928,1717,1691,1556,1447,1437,1383$, $1282,1259,1138,1059,914 .{ }^{1} \mathrm{H}$ NMR $\left(400 \mathrm{MHz}, \mathrm{CDCl}_{3}\right): \delta 8.81(\mathrm{br}, 1 \mathrm{H}, \mathrm{NH}), 7.52(\mathrm{~d}, 2 \mathrm{H}, J=7.6, \mathrm{~Hz}), 7.38$ $(\mathrm{t}, 2 \mathrm{H}, J=8.0 \mathrm{~Hz}), 7.28(\mathrm{t}, 1 \mathrm{H}, J=7.2 \mathrm{~Hz}), 6.90(\mathrm{~d}, 1 \mathrm{H}, J=3.6 \mathrm{~Hz}), 3.91\left(\mathrm{~s}, 3 \mathrm{H}, \mathrm{OCH}_{3}\right), 2.84\left(\mathrm{~s}, 6 \mathrm{H}, 2 \mathrm{CH}_{3}\right) .{ }^{13} \mathrm{C}$ $\operatorname{NMR}\left(100 \mathrm{MHz}, \mathrm{CDCl}_{3}\right): \delta 160.4,134.9,128.34,128.28,128.15,126.4,122.0,120.4,114.7,51.3,44.3$ (HRMS $=244.1206$, requires $\mathrm{C}_{14} \mathrm{H}_{16} \mathrm{~N}_{2} \mathrm{O}_{2}$ 244.1212). 


\subsubsection{4-Phenyl-1 $H$-1,2,3-Triazole 10a}

White solid, mp. $146-148{ }^{\circ} \mathrm{C}$ (lit.mp 147-147.4 ${ }^{\circ} \mathrm{C}$, Zhang, Kung, \& Yang, 2010).

\subsubsection{Ethyl-1H-1,2,3-Triazole-4-Carboxylate $\mathbf{1 0 b}$}

Colorless solid, mp.103-104 ${ }^{\circ} \mathrm{C}$ (lit.mp 102-103 ${ }^{\circ} \mathrm{C}$, Avat-Arman \& Khojasteh, 2009).

\subsubsection{4-Allyl-5-Phenyl-4H-1,2,3-Triazole 13}

Colorless oil, $\left(\mathrm{R}_{\mathrm{f}}\right.$ 0.85, EtOAc: petroleum b.p. $\left.60-80{ }^{\circ} \mathrm{C}, 1: 3 \mathrm{v} / \mathrm{v}\right) . \mathrm{MS}: m / z(\%)=185\left(\mathrm{M}^{+}, 10\right), 157(20), 116$ (100). IR (KBr, cm $\left.{ }^{-1}\right): 3081,3032,2924,1495,1454,1275,1076,995,925,754,699 .{ }^{1} \mathrm{H}$ NMR (400 MHz, $\left.\mathrm{CDCl}_{3}\right): \delta 7.56-7.28(\mathrm{~m}, 5 \mathrm{H}), 5.88-5.78(\mathrm{~m}, 1 \mathrm{H}), 5.22(\mathrm{t}, 2 \mathrm{H}, J=7.2 \mathrm{~Hz}), 3.88(\mathrm{t}, 1 \mathrm{H}, J=7.2 \mathrm{~Hz}), 2.72-2.61(\mathrm{~m}$, $2 \mathrm{H}) .{ }^{13} \mathrm{C}$ NMR $\left(100 \mathrm{MHz}, \mathrm{CDCl}_{3}\right): \delta 135.2,132.5,129.0,128.1,127.3,120.3,119.3,39.8,37.5(\mathrm{HRMS}=$ 185.0947, requires $\mathrm{C}_{11} \mathrm{H}_{11} \mathrm{~N}_{3}$ 185.0940).

\section{Results and Discussion}

\subsection{Synthesis}

1-Alkyl substituted-1,2,3-triazoles 4a-f were prepared from terminal alkynes, sodium azide and alkyl halides using $\mathrm{CuSO}_{4} \cdot 5 \mathrm{H}_{2} \mathrm{O}$ and $\mathrm{Na}$-ascorbate in ter-butanol/water mixture solvent after stirring at room temperature for 3-12 hrs some of which are new compounds (click chemistry, Liu \& Reiser, 2011). Condensation of 4a-d with dimethylformamide dimethyl acetal (DMFDMA) in a microwave oven (M.W.) at $150{ }^{\circ} \mathrm{C}$ for $3 \mathrm{~min}$. produced 1alkenyl substituted-1,2,3-triazoles 5a-d. Coupling of 4a with the appropriate aromatic diazonium chloride in ethanol and sodium acetate produced 1-arylhydrazono-1-(4-phenyl-1,2,3-trizole-1-yl)propan-2-ones 6a-c (Scheme 2). The UV spectrum of these compounds shows absorption maxima in the region 248-312 nm (Table $1)$.
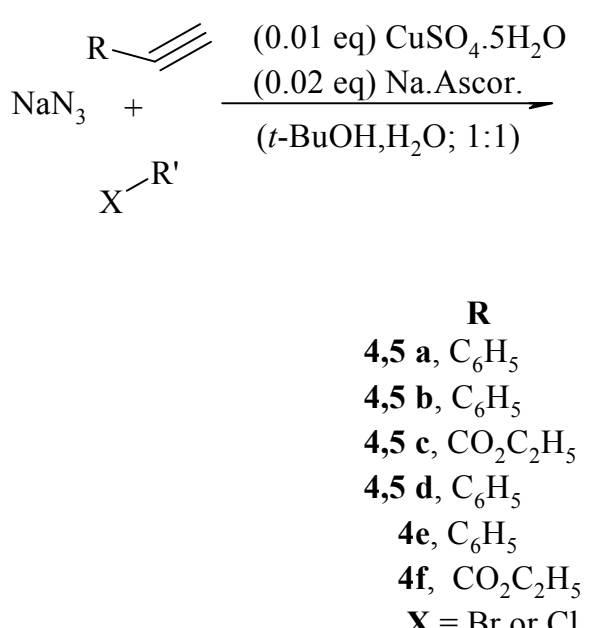
$\mathbf{X}=\mathrm{Br}$ or $\mathrm{Cl}$<smiles>[R]c1nnn([R])c1[14CH3]</smiles>

$\mathbf{R}^{\prime}$

$\mathrm{CH}_{2} \mathrm{COCH}_{3}$ $\mathrm{CH}_{2} \mathrm{CO}_{2} \mathrm{CH}\left(\mathrm{CH}_{3}\right)_{2}$ $\mathrm{CH}_{2} \mathrm{COC}_{6} \mathrm{H}_{5}$ $\mathrm{CH}_{2} \mathrm{CO}_{2} \mathrm{CH}_{3}$ $\mathrm{CH}_{2} \mathrm{CH}=\mathrm{CH}_{2}$ $\mathrm{CH}=\mathrm{CHCO}_{2} \mathrm{C}_{2} \mathrm{H}_{5}$<smiles>CC(=O)/C(=N/N[14CH3])n1cc(-c2ccccc2)nn1</smiles>

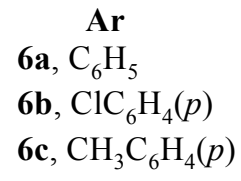

Scheme 2. Synthesis of 1-substituted-1,2,3-triazoles 4a-f, 5a-d and 6a-c

The structure of compounds 4a-f, 5a-d and 6a-c were well established based on full data of ${ }^{1} \mathrm{H},{ }^{13} \mathrm{C}$ and $2 \mathrm{D}$ NMR, GC-MS, LCMS (see Experimental section) and moreover X-ray crystal structures of compounds $\mathbf{4 f}, \mathbf{5 a}$ and $\mathbf{6 c}$ were determined (Figure 1). 


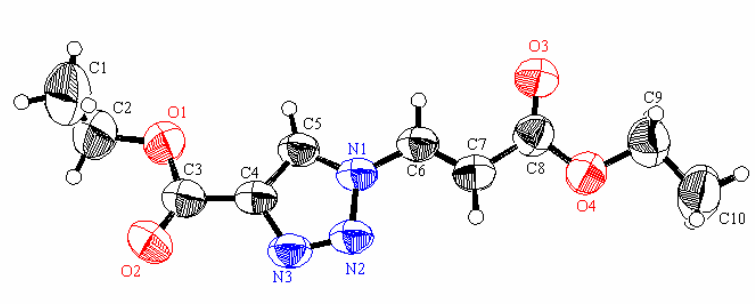

$4 \mathbf{f}$

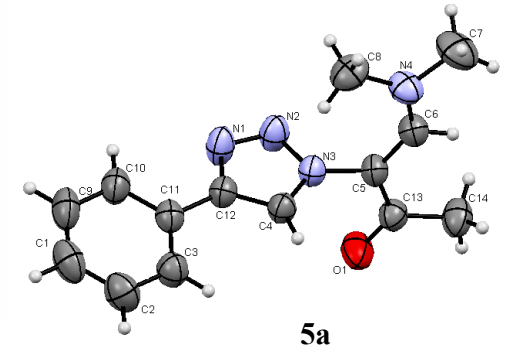

$5 \mathbf{a}$

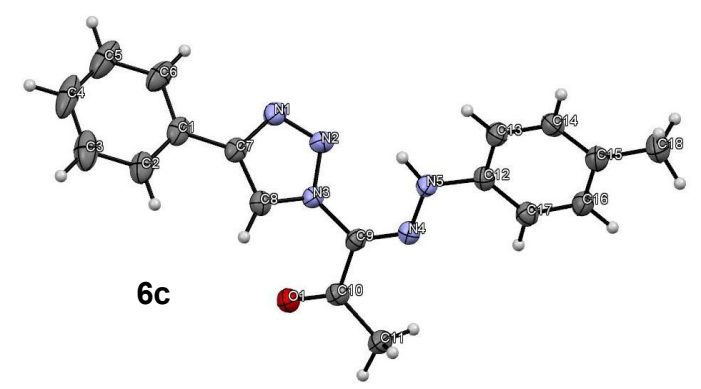

Figure 1. ORTEP drawing of compounds $\mathbf{4 f}, 5 \mathbf{a}$ and $\mathbf{6 c}$

\subsection{Photolysis}

Irradiation of 1-alkenyl substiuted-1,2,3-triazoles 5a-d using $16 \mathrm{~W}$ low pressure mercury arc-lamp for 16 hrs produced 3-dimethylamino-1H-pyrrole derivatives 9a-d, in 34-38\% yield together with 4-phenyl or (4ethoxycarbonyl)- $1 H-1,2,3$-triazoles $\mathbf{1 0 a}, \mathbf{b}$ in $25-35 \%$ yield. The formation of these photoproducts can be explained through extrusion of $\mathrm{N}_{2}$ molecule to form the corresponding 1,3-diradical intermediate 7 which cyclized to $\mathbf{8}$ followed by 1,3H-shift produced $\mathbf{9 a - d}$. Formation of 10a,b can be explained by photo-hydrolysis of N1-C bond (Scheme 3).

The structure of the new pyrrole derivatives 9a-d were established based on full data ${ }^{1} \mathrm{H},{ }^{13} \mathrm{C}-\mathrm{NMR}$, GC-MS and X-ray crystal structures of compounds $\mathbf{9 a , c , d}$ (Figure 2).

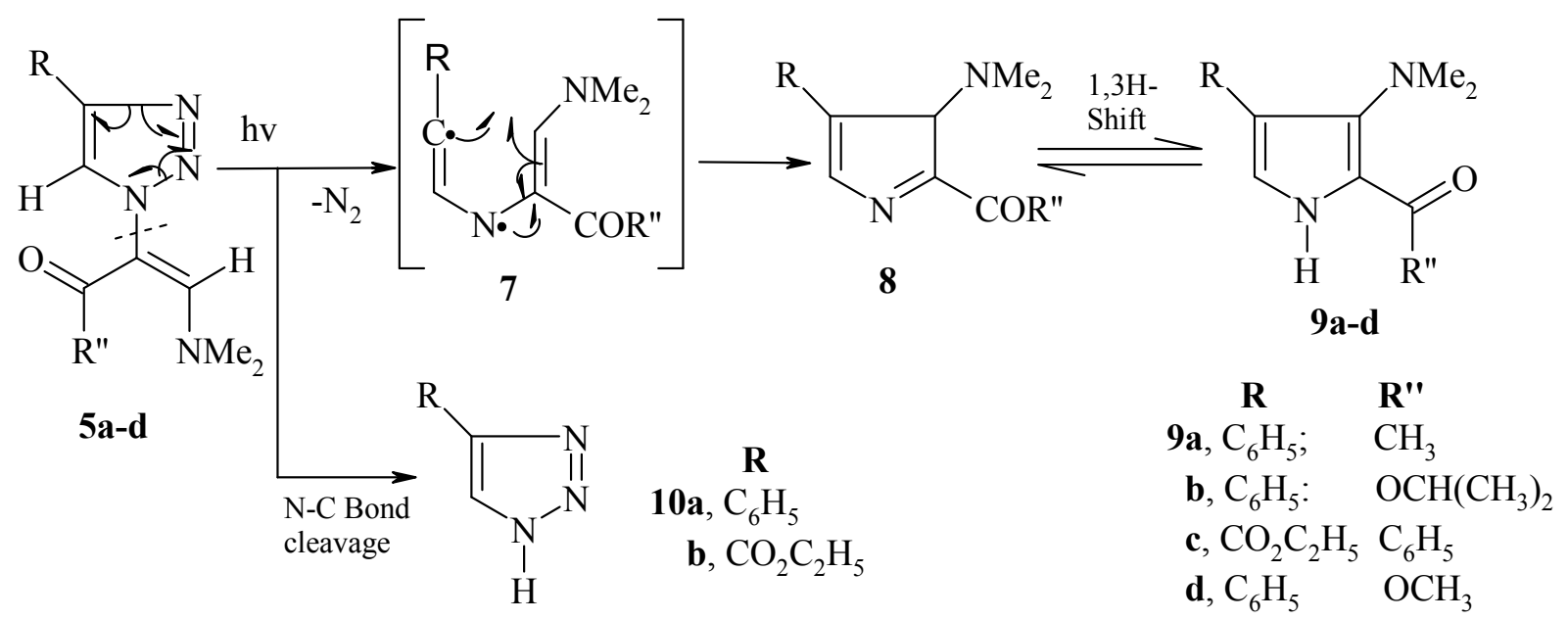

10a,b

Scheme 3. Photoproducts of compounds 5a-d 

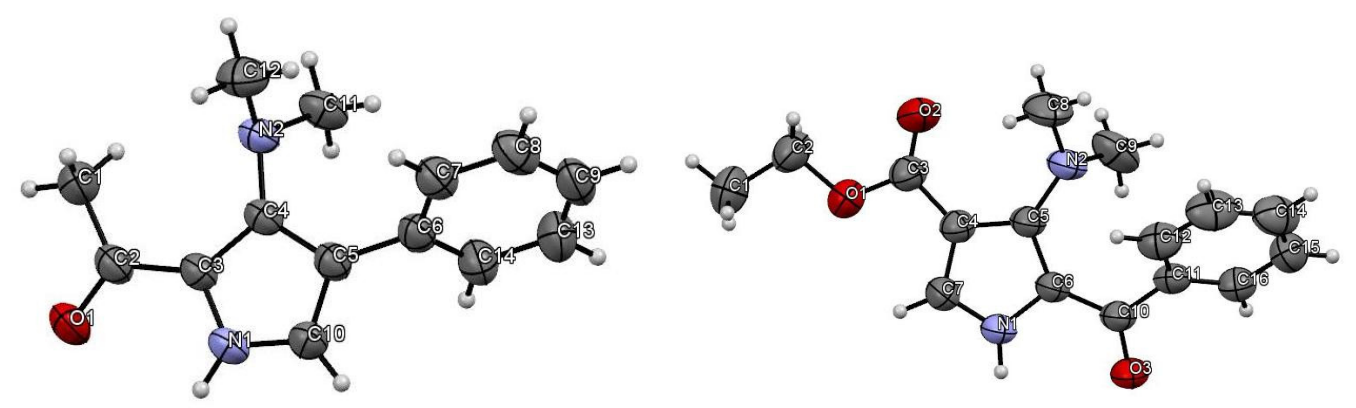

$9 a$

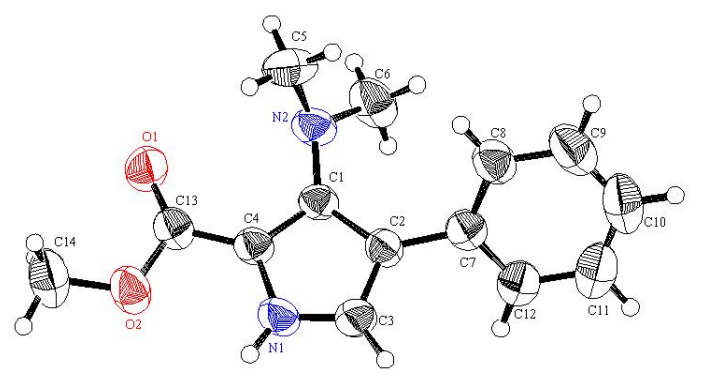

9c

9d

Figure 2. ORTEP drawing of compounds $9 \mathbf{a}, \mathbf{9 c}$ and $\mathbf{9 d}$

Irradiation of compound $\mathbf{4 e}$ under the same conditions produced 4-allyl-5-phenyl-4H-1,2,3-triazole $\mathbf{1 3}$ in $38 \%$ yield through 1,5-allyl shift, and not the expected pyrrole 11 (Scheme 4). The structure of photoproduct $\mathbf{1 3}$ was suggested by 2D NMR, HSQC, H,H-COSY and HMBC, 2-D experiments (Figure 3).

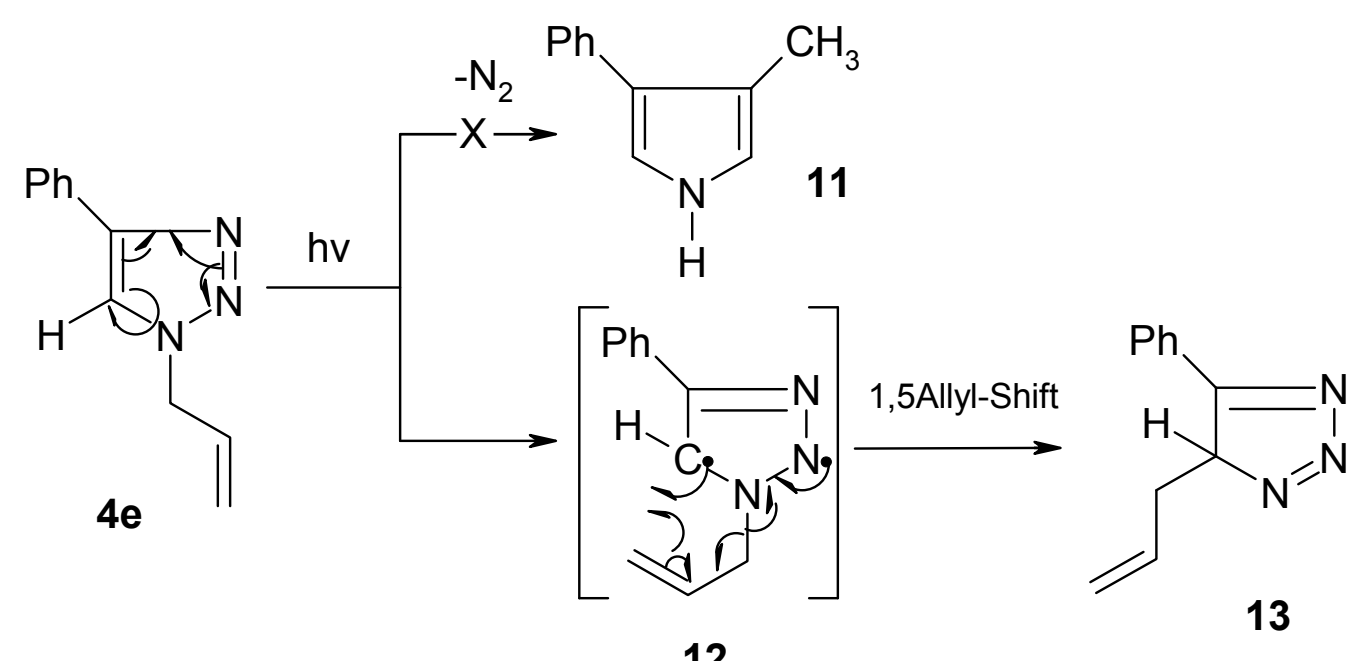

Scheme 4. Photoproduct of compound $4 \mathbf{e}$ 

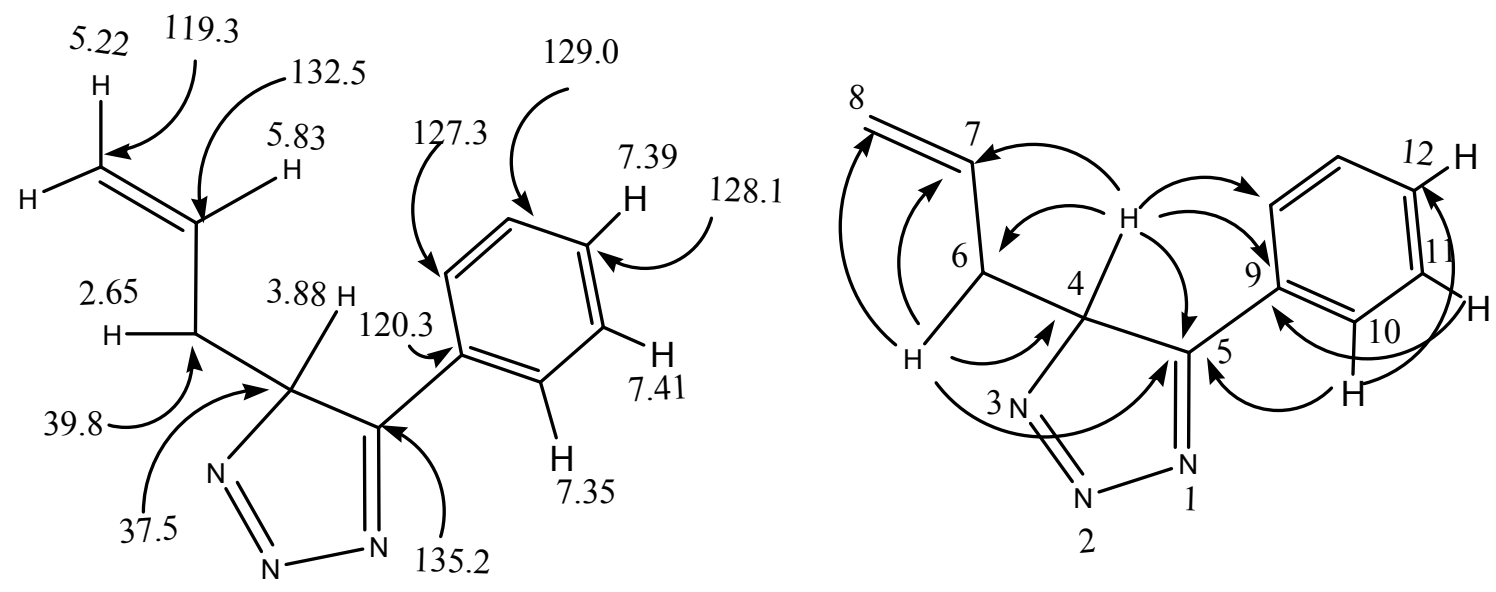

Figure 3. Important HMBC, H-C correlation of compound $\mathbf{1 3}$

Irradiation of compounds $\mathbf{4 f}$ (trans) under the same condition afforded formation of photo-stachionary $E-Z$ photo-isomerization mixture (trans/cis, $60: 40 \%$ ) isomers of $\mathbf{4 f}$ (Scheme 5). Moreover irradiation of the isolated cis isomers $\mathbf{4 f}$ yielded trans/cis, 60: 40\% mixture. The two isomers were separated and fully characterized by ${ }^{1} \mathrm{H}$, ${ }^{13} \mathrm{C}-\mathrm{NMR}$ (see Experiment section).

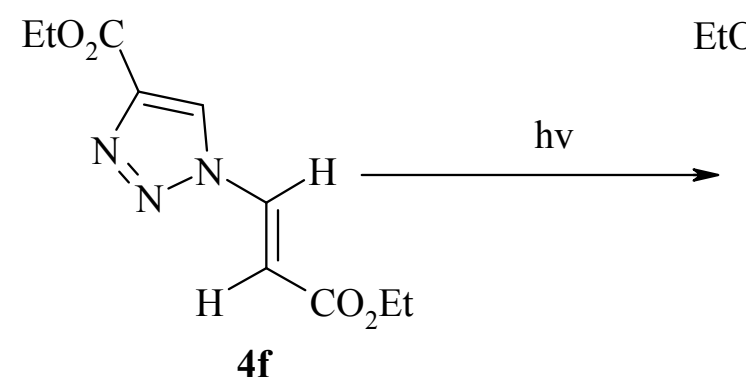

Trans<smiles>CCOC(=O)/C=C/n1cc(C(=O)OCC)nn1</smiles>

(Trans : cis)

$60: 40$

Scheme 5. Trans/Cis photoisomerisation of compound $\mathbf{4 f}$

Irradiation of 1-arylhydrazono-1,2,3-triazole derivatives 6a-c in an attempt to prepare the corresponding 1anilino-imidazole derivatives 14a-c through loss of molecular nitrogen did not produce any photoproducts (Scheme 6). Table 1 shows the absorption maxima $\left(\lambda_{\max }\right)$ and \% yield of photoproducts $4 \mathbf{e}, \mathbf{f}$ and $\mathbf{5 a - d .}$<smiles></smiles>

Scheme 6. Photolysis of compounds 6a-c 
Table 1. Photoproducts of compounds 4e,f and 5a-d

\begin{tabular}{ccccc}
\hline Substrat & $\lambda$ max & $\begin{array}{c}\text { Irradiation } \\
\text { condition }\end{array}$ & $\begin{array}{c}\text { Irradiation } \\
\text { time }(\mathrm{h})\end{array}$ & $\begin{array}{c}\text { Photoproducts } \\
\text { and Yield \% }\end{array}$ \\
\hline $\mathbf{4 e}$ & 248 & $16 \mathrm{~W}$ & $16 \mathrm{~h}$ & $\mathbf{1 3}(38)$ \\
$\mathbf{4 f}$ & 260 & $16 \mathrm{~W}$ & $16 \mathrm{~h}$ & $\mathbf{4 f}(60$ trans: 40 cis $)$ \\
$\mathbf{5 a}$ & 287 & $16 \mathrm{~W}$ & $16 \mathrm{~h}$ & $\mathbf{9 a}(36), \mathbf{1 0 a}(35)$ \\
$\mathbf{5 b}$ & 296 & $16 \mathrm{~W}$ & $16 \mathrm{~h}$ & $\mathbf{9 b}(34), \mathbf{1 0 a}(28)$ \\
$\mathbf{5 c}$ & 312 & $16 \mathrm{~W}$ & $16 \mathrm{~h}$ & $\mathbf{9 c}(35), \mathbf{1 0 b}(25)$ \\
$\mathbf{5 d}$ & 284 & $16 \mathrm{~W}$ & $16 \mathrm{~h}$ & $\mathbf{9 d}(38), \mathbf{1 0 a}(30)$ \\
\hline
\end{tabular}

\section{Conclusions}

1-alkenyl substituted-1,2,3-triazoles 5a-d were photochemically converted into 3-dimethylamino- $1 H$-pyrrole derivatives 9a-d together with 4-phenyl or (4-ethoxycarbonyl)-1H-1,2,3-triazoles 10a,b. The photochemical behavior of 1-substituted-1,2,3-triazoles 5a-d is similar to those of benzotriazoles (Al-Jalal, Al-Awadi, Ibrahim, \& Elnagdi, 2011). However 1-allyl-4-phenyl-1 $H$-1,2,3-triazole 4e produced upon irradiation 4-allyl-5-phenyl4H-1,2,3-triazole 13, while trans 1-(2-ethoxycarbonyl-vinyl)-1H-1,2,3-triazole-4-carboxylic acid ethyl ester $\mathbf{4 f}$ produced trans/cis photo-isomerization mixture.

\section{Supplementary Material}

Crystallographic data of (excluding structure factors) for the structure in this paper have been deposited with the Cambridge Crystallographic Data Centre as supplementary publication nos. CCDC 928598 (4f), CCDC 928127 (5a)and CCDC 928599 (6c), CCDC 929700 (9a), CCDC 929701 (9c) and CCDC 939635 (9d). Copies of the data can be obtained, free of charge, on application to CCDC, 12 Union Road, Cambridge CB2 1EZ, UK, fax: +44-(0)1223-336033 or e-mail: deposit@ccdc.cam.ac.uk.

\section{Acknowledgements}

The support of the University of Kuwait received through research grant \# SC 04/08 and the facilities of ANALAB/SAF (grants no. GS01/01, GS02/01, GS03/08) are gratefully acknowledged.

\section{References}

Al-Awadi, H., Ibrahim, M., Ibrahim, Y., \& Al-Awadi, N. (2008). Gas-phase thermolysis of benzotriazole derivatives. Part 4: Pyrolysis of 1-acylbenzotriazole phenylhydrazones. Intersting direct routes toward $\mathrm{N}$-aminobenzimidazoles. J. Heterocyclic Chem., 45, 723-727. http://dx.doi.org/10.1002/jhet.5570450314

Al-Jalal, N., Al-Awadi, N., Ibrahim, M., \& Elnagdi, M. (2011a). The photochemistry of 1-alkenyl-substituted-1,2,3-triazole leading to formation of indole and fused indole derivatives. Arkivoc, 2011(10), 299-308. http:/dx.doi.org/10.3998/ark.5550190.0012.a24

Al-Jalal, N., Ibrahim, M., Al-Awadi, N., \& Elnagdi, M. (2011b). The photochemistry of benzotriazol derivatives. Part 2: Photolysis of 1-substituted benzotriazole arylhydrazone: New route to phenanthridin-6-yl-2-phenyldiazine.

Molecules, $\quad$ 16, $8257-8263$. http://dx.doi.org/10.3390/molecules 161210256

Avat-Arman, T., \& Khojasteh, K. (2009). One-pot microwave-assisted solvent free synthesis of simple alkyl 1,2,3-triazole-4-carboxylates by using trimethylsilylazide. $J$. Heterocyclic Chem., 46, 131-133. http://dx.doi.org/10.1002/jhet.36

Bellina, F., \& Rossi, R. (2006). Syntheis and biological activity of pyrrole, pyrroline, and pyrrolidinederivatives with two aryl groups on adjacent positions. Tetrahedron, 62, 7213-7256. http://dx.doi.org/10.1016/j.tet.2006.05.024

Boyer, J. H., \& Selvarajan, R. (1969). Photolysis of vic-triazole. Tetrahedron Lett., 10, 47-50. http://dx.doi.org/10.1016/S0040-4039(01)97649-x

Burgess, E. M., Carithers, R., \& McCullagh, L. (1968). Photochemical decomposition of 1H-1,2,3-triazole derivatives. J. Am. Chem. Soc., 90, 1923-1924. http://dx.doi.org/10.1021/ja01009a056 
Dib, H., Al-Awadi, N., Ibrahim, Y., \& El-Desoqui, O. (2003). Gas-phase thermolysis of benzotriazole derivatives. Part 2: Synthesis of benzimidozo[1,2-b]cinnolines, a novel heterocyclic ring system, by pyrolysis of benzotriazole derivatives. Kinetic and mechanistic study. Tetrahedron, 59, 9455-9464. http://dx.doi.org/10.1016/j.tet.2003.09.009

Dib, H., Al-Awadi, N., Ibrahim, Y., \& El-Desoqui, O. (2004). Gas-phase thermolysis of benzotriazole derivatives. Part 1-synthesis of $\alpha-\mathrm{N}(1)$ - and $\mathrm{N}(2)$-benzotriazolyl ketones and kinetic and mechanism of their gas-phase pyrolysis. J. Phys. Org. Chem., 17, 267-272. http://dx.doi.org/10.1002/poc.717

Katritzky, A. R., Lan, X., Yang, J., \& Denisko, O. (1998). Preparation and synthetic utility of N-substituted benzotriazole. Chem. Rev., 98, 409. http://dx.doi.org/10.1021/cr941170v

Kidwai, M., \& Jain, A. (2011). Regioselective synthesis of 1,4-disubstituted triazoles using bis[(L)-prolinato-N,O]Zn complex as an efficient catalyst in water as a sole solvent. App. Organom. Chem., 25, 620-625. http://dx.doi.org/10.1002/aoc. 1816

Kumar, D., Patel, G., \& Reddy, V. B. (2009). Green and expeditious synthesis of 1,4-disubstituted 1,2,3-triazoles from terminal acytelene and in situ generated $\alpha$-azido ketones. Synlett, 3, 399-402. http://dx.doi.org/10.1055/s-0028-1087556

Liebscher, J., Knoll, A., Ushmaev, A., Rolfs, A., Lohmann, D., Faust, G., . . Scharfenberg, P. (1992). Ger. East, DD 298915 A5 19920319.

Liu, M., \& Reiser, O. (2011). A copper (1) isonitrile complex as a heterogeneous catalyst for azide-alkyne cycloaddition in water. Org. Lett., 13, 1102-1105. http://dx.doi.org/10.1021/ol103134c

Mäerky, M., Schmid, H., \& Hansen, H. (1979). Photoreaction of 1-alkylbenzotriazoles. Helv Chim. Acta, 62, 2129-2153. http://dx.doi.org/10.1002/hlca.19790620710

Michell, G., \& Rees, C. W. (1987). Photolysis of 1-aryl-1,2,3-triazoles; rearrangement via 1H-azirines. J. Chem. Soc., Perkin Trans, 1, 413-422. http://dx.doi.org/10.1039/p19870000413

Ogata, Y., Takaji, K., \& Hayashi, E. (1977). Photochemical Dimroth rearrangement of 1,4-diphenyl-5-aminoand 4-phenyl-5-anilino-1,2,3-triazoles. Bull. Chem. Soc. Jpn., 50, 2505-2506. htt://dx.doi.org/10.1246/bcsj.50.2505

Rochais, C., Lisowski, V., Dallemagne, P., \& Rault, S. (2004). First synthesis of methyl 3-amino-4-(het)aryl-1H-pyrrole-2-carboxylates as useful scaffolds in medicinal chemistry. Tetrahedron, 60 , 2267-2270. http://dx.doi.org/10.1016/j.tet.2004.01.019

Silvarajan, R., \& Boyer, J. H. (1972). Photo-and thermal elimination of nitrogen from 4-phenyl-and 4,5-diphenyl-1,2,3-triazole. J. Heterocyclic Chem., 9, 87-90. http://dx.doi.org/10.1002/jhet.5570090114

Wender, P. A., \& Cooper, C. B. (1986). The photochemistry of 1-alkenylbenzotriazoles. Methodology for the synthesis of indoles. Tetrahedron, 42, 2985-2991. http://dx.doi.org/10.1016/S0040-4020(01)90589-7

Wender, P. A., \& Cooper, C. B. (1987). Indole synthesis based on triazole photochemistry: Total synthesis of 7-methoxymitosene. Tetrahedron Lett., 28, 6125-6128. http://dx.doi.org/10.1016/S0040-4039(00)61825-7

Zhang, W., Kung, C., \& Yang, Q. (2010). Palladium-catalyzed one-pot synthesis of 4-aryl-1H-1,2,3-triazoles from anti-3-aryl-2,3-dibromopropanoic acids and sodium azide. Synthesis, 2, 283-287. http://dx.doi.org/10.1055/s-0029-1217097

\section{Copyrights}

Copyright for this article is retained by the author(s), with first publication rights granted to the journal.

This is an open-access article distributed under the terms and conditions of the Creative Commons Attribution license (http://creativecommons.org/licenses/by/3.0/). 